\title{
The BRITE satellite and Delta Scuti Stars: The Magnificent Seven
}

\author{
M. Breger \\ Institut für Astronomie, Türkenschanzstrasse 17, 1180 Vienna, Austria
}

\section{Abstract}

This paper examines the prospect of observing $\delta$ Scuti variables with BRITE. In particular, some of the astrophysical questions, which can be investigated, are discussed together with the methods to be applied. Finally, the seven bright stars suitable for BRITE are presented.

\section{Introduction}

The $\delta$ Scuti stars are pulsators situated in the classical cepheid instability strip on the main sequence or moving from the main sequence to the giant branch. In general, the period range is limited to between $0.02 \mathrm{~d}$ and $0.25 \mathrm{~d}$. This limit provides a good separation from the neighboring or overlapping groups of pulsators in the Hertzsprung-Russell Diagram, such as roAp, $\gamma$ Dor and RR Lyrae stars.

Regrettably, one should not regard this simple definition and description to be complete. We must consider two additional astrophysical situations: (i) the evolved Pop. II stars inside the classical instability strip with $\delta$ Scuti-like periods (SX Phe stars, unobservable with BRITE), and (ii) the massive stars evolving through the instability strip.

The massive $\left(\mathcal{M}>2 \mathcal{M}_{\odot}\right)$ stars evolving from the blue main sequence towards the giant region cross the instability strip on nearly horizontal tracks in the Hertzsprung-Russell Diagram. The evolutionary state identifies them as $\delta$ Scuti stars. But due to their high mass and luminosity, the periods are longer than those of an average $\delta$ Scuti star and may get as large as $1 \mathrm{~d}$. Consequently, in period they overlap the RR Lyrae stars, which are in the postgiant stage of evolution and have low masses below $1 M_{\odot}$. It has been proposed (Breger 2000) that these long-period $\delta$ Scuti stars be distinguished from RR Lyrae stars by considering the size of stellar rotation. The suggestion is based on the observation by Petersen, Carney \& Latham (1996), who point out that RR Lyrae stars show no detectable rotation ( $\left.v \sin i \leq 10 \mathrm{~km} \mathrm{~s}^{-1}\right)$. 
$\delta$ Scuti stars can also be found among pre-main sequence stars. Examples are the pulsators in the young clusters NGC 2264 (Breger 1972), IC 4996 and NGC 6530 (Zwintz \& Weiss 2006).

Some $\delta$ Scuti stars are (pure) radial pulsators, while the majority pulsate with a large number of nonradial $p$ modes simultaneously. The nonradial pulsations of $\delta$ Scuti stars found photometrically are low-degree $(\ell \leq 3)$ and low-order ( $\mathrm{n}=0$ to 7$) \mathrm{p}$ modes, while spectroscopic studies have confirmed the presence of high-degree nonradial modes with $\ell$ up to 20 (e. g., for the star $\tau$ Peg, Kennelly et al. 1998).

The $\delta$ Scuti stars represent a transition between the cepheid-like largeamplitude radial pulsation of the classical instability strip and the ocean of nonradial pulsation occurring in the hot half of the Hertzsprung-Russell Diagram. Many excited modes show photometric amplitudes in excess of 0.001 mag, which makes it possible to study these stars photometrically. The position of $\delta$ Scuti stars on and slightly above the main sequence permits the asteroseismological comparison between oscillation data and stellar models in a region where the basic stellar structure is regarded as relatively well known.

\section{Some reasons for the expected success of BRITE data}

The BRITE project cannot be in the same league as large satellite missions such as COROT or GAIA. However, this situation could lead to some important advantages as well:

(i) BRITE can focus on a few specific astrophysical questions and objects. For large missions, a number of scientific compromises have to accepted to ensure a return of the investment and to satisfy a large, sometimes diverse, community.

(ii) Since BRITE has to measure bright stars, the stellar parameters are known. This makes astrophysical interpretations of the results much easier and more reliable.

(iii) Because of the relative 'smallness' of the mission, data reduction and the scientific analyses can be very fast.

(iv) Huge quantities of data require special methods of data analysis including application of 'blind' methods. The BRITE data can avoid these blind methods and can be analyzed by experienced astronomers examining the data in detail at all stages of the reduction process. I regard this as very important: BRITE might lead to data unparalleled in their excellence. 


\section{Why study Delta Scuti stars with BRITE?}

Asteroseismology relies on the comparison between observed and model frequencies. To achieve this aim, extensive and high-quality observational data are required. Let us illustrate this general statement with three out of many possible examples.

Frequency range of excited regions: direct implications for realistic models

The range of excited frequencies of a star is an important tool for modeling since these limits are very sensitive to effects such as convection and abundance.

\section{Low-frequency region: gravity modes} Observed so far: None or 1 millimag amplitude

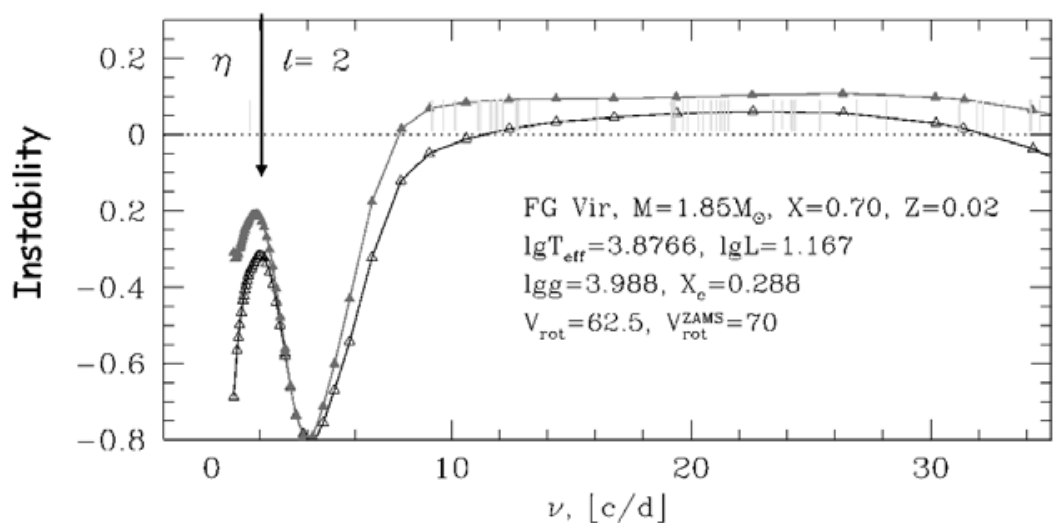

Figure 1: A pulsation excitation model computed by A. A. Pamyatnykh for a typical evolved $\delta$ Scuti star. The parameter, $\eta$, denotes the amount of instability. Note the low-frequency peak, still situated in the stable region. However, some change in model parameters could shift the peak beyond the instability line in order to match observed low-frequency pulsation.

Fig. 1 shows a pulsation excitation model for FG Vir. We note that there exists a low-frequency peak near $2 \mathrm{c} / \mathrm{d}$. In this model, the peak is still in the stable (unexcited) region. However, some changes in the model can push the peak to instability. This would then become an observable g-mode. There exists some evidence that these have already been detected at low amplitude in a few $\delta$ Scuti stars.

The main pulsation of the star FG Vir occurs around $20 \mathrm{c} / \mathrm{d}$. However, the star also shows a number of high-frequency pulsation modes at frequencies 
above $40 \mathrm{c} / \mathrm{d}$ with small, statistically significant amplitudes of pulsation below one millimag. The observed high-frequency cutoff allows the models to be finetuned, especially with respect to the convection treatment. The high-frequency amplitudes are very low, requiring extensive observations, as could be provided by BRITE.

\section{Photometric mode identification: the case for two filters}

Let us turn to the identification of individual pulsation modes. For most asteroseismic applications, the frequency information of a particular pulsation mode is not sufficient: the quantum numbers of pulsation $(n, \ell$, and $m)$ need to be known. Pulsation mode identification can be very complex. A powerful tool is provided by the phase differences and amplitude ratios between the measurements made through different passbands. For $\delta$ Scuti stars, the two Strömgren filters, $v$ and $y$, are often used. Here the phase differences (i.e., time shifts) between the two passbands are a strong indicator of the $\ell$ values, the number of node lines on the stellar surface.

Fig. 2 illustrates the method for the star 44 Tau. Since BRITE is planned to measure with two filters, the photometric mode identification method can be applied to $\delta$ Scuti stars.

Amplitude variability and close frequencies

Amplitude variability is a common property of pulsating stars. For RR Lyrae stars, this phenomenon is known as the Blazhko Effect since it was noticed by Blazhko (1907) in the RR Lyrae star RW Dra. However, a large number of different types of pulsators in different stages of evolution also show amplitude and phase variability on non-evolutionary time scales. Examples are classical cepheids (e.g., Breger 1981), sdB stars (e.g., Kilkenny et al. 1999), $\beta$ Cephei stars (Lehmann et al. 2001), White Dwarfs (e.g., Handler et al. 2003), and $\delta$ Scuti stars (Breger \& Bischof 2002)

Regrettably, the physical origin of these variations is not yet known. One of the reasons is the lack of extensive observational data to distinguish between different hypotheses. The excellent observational coverage, which can be provided by BRITE, makes these stars an excellent tool to study amplitude variability: In the Fourier spectrum of short data sets, two close frequencies beating with each other appear as a single frequency with variable amplitude and phase. For long data sets with sufficient frequency resolution, two peaks will be shown. In the case of a single frequency with a real variable amplitude and/or phase, two (or more) peaks also occur. Consequently, the appearance of two peaks in the Fourier spectrum does not prove the existence of two separate 


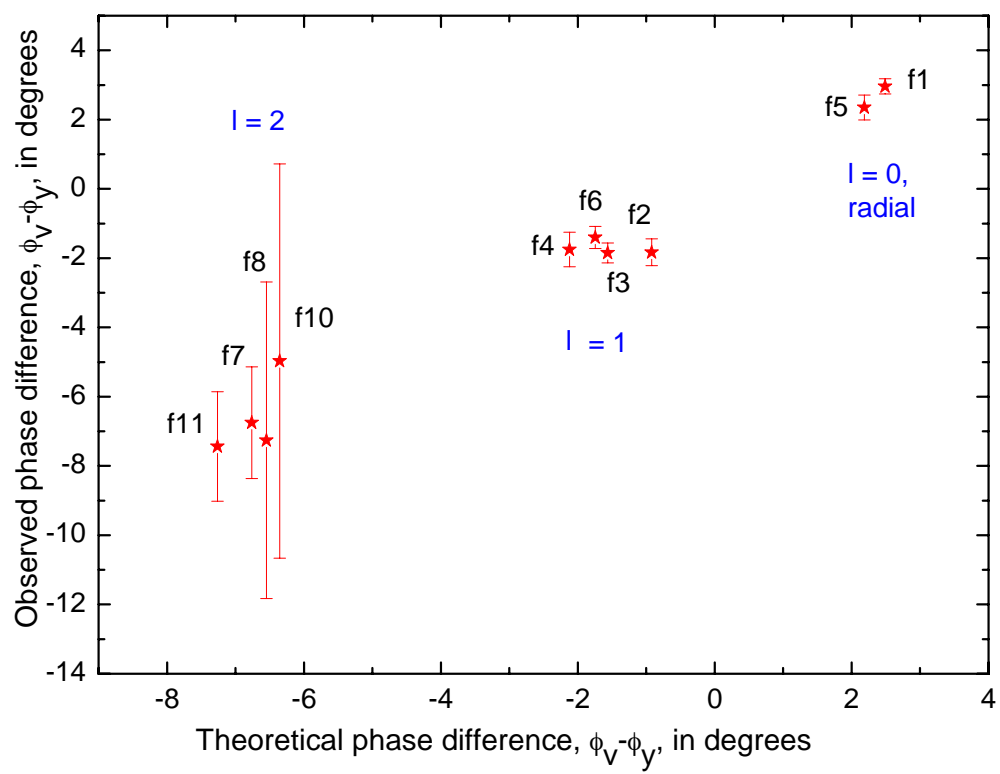

Figure 2: The diagram shows that the observed phase differences between the $v$ and $y$ passbands can successfully identify the pulsational $\ell$ values by comparing the observations with phase differences predicted from models. The star selected is $44 \mathrm{Tau}$. The observational error bars are very small for $\ell=0$ and 1 modes, while the identification of the $\ell=2$ modes is less certain due to their small photometric amplitudes. The model for 44 Tau is taken from Lenz et al. (2007).

modes, while the absence of a double peak does not exclude the possibility. This is a fact well recognized in the literature.

Fortunately, beating between two (or more) frequencies produces amplitude and phase variations which are mathematically related. The most extreme and easily recognizable situation occurs when two close frequencies have the same amplitude: this leads to a half a cycle phase shift at the time of minimum amplitude of the visible (single) frequency. Even when the amplitudes differ, beating has a specific signature in the amplitude of phase shifts of an assumed single frequency. In particular, the largest phase change (of an assumed single frequency) occurs at the time of minimum amplitude.

Therefore, it is possible to separate beating from true amplitude variability by studying the relationship between the amplitude and phase variations of an assumed single frequency. In practice, large differences in the amplitudes of the frequencies beating with each other, observational noise, as well as short 


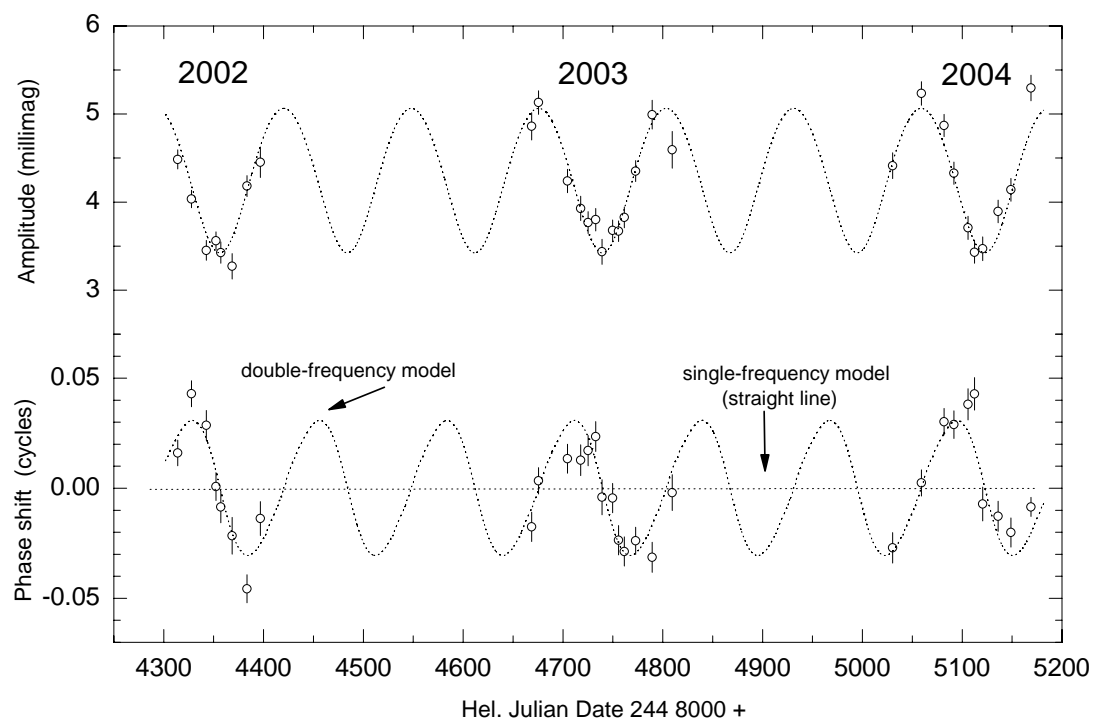

Figure 3: This diagram shows that the observed amplitude and phase variations of the $12.154 \mathrm{c} / \mathrm{d}$ frequency in FG Vir are caused by beating between two close frequencies. The open circles represent the observations, while the dotted line is the two-frequency fit. The variations can be explained by two independent frequencies separated by $0.0078 \mathrm{c} / \mathrm{d}$ and an amplitude ratio of 0.19 . Note the excellent agreement between the different years.

(relative to the beat period) data sets may make the recognition difficult.

The method consists of obtaining an extensive photometric data base, subdividing the data into short time bins and calculating the amplitude and phase of the assumed optimum single frequency for each time bin. Furthermore, if other pulsation frequencies are also present, they need to be corrected for.

The test between true amplitude variability and beating between close frequencies has already been applied by us to three $\delta$ Scuti variables. The variability occurs in radial as well nonradial modes. Let us examine a radial mode at $12.154 \mathrm{c} / \mathrm{d}$ in the star FG Vir, for which extensive photometric data are available (Breger et al. 2005). Fig. 3 shows the variations in amplitude and phase for the three years.

The variations are similar in all three years with a beat period of $128 \mathrm{~d}$. Another important result is that the phase changes are coupled to the amplitude changes. In particular, minimum amplitude occurs at the time of 'average' phase and the time of most rapid phase change. As already mentioned earlier, this is an important signature of beating between two close frequencies. The visual 
result is confirmed by a two-frequency model with the optimum parameters of frequency, amplitude and phase determined by PERIOD04 (Lenz \& Breger 2005). An excellent agreement is obtained for both the amplitude and phase changes with the two-frequency model. It should be noted that the test was successful even with a second mode of small amplitude and phase shifts under 0.05 cycles. If we extend the analysis to include the 1995 data, we also find an excellent agreement between the predicted and observed amplitudes and phases of the earlier measurements.

We conclude that in FG Vir the amplitude and phase variations of the mode at $12.154 \mathrm{c} / \mathrm{d}$ can be explained by the beating of two close frequencies. The analysis has been repeated for a number of other pulsation modes in FG Vir and $\mathrm{BI} \mathrm{CMi}$. The results are the same: the amplitude and phase variability is caused by beating of close frequencies.

It has, therefore, been shown that beating between close frequencies is responsible for at least some of the observed amplitude and phase variability. However, can it explain all observed cases? Our presently unpublished data for $4 \mathrm{CVn}$ suggests that an additional effect may be evolved, especially for long-term variations just short of the evolutionary time scales. The accurate measurements with BRITE should provide important new results to help decide whether two or more effects are responsible.

\section{The Magnificent Seven}

Seven known $\delta$ Scuti stars are bright enough to be observed with BRITE. Due to the brightness (!) their photometric variations have not in all cases been well-studied from the ground and the pulsation information given in the table below may not be reliable.

Acknowledgments. This investigation has been supported by the Austrian Fonds zur Förderung der wissenschaftlichen Forschung.

\section{References}

Blazhko, S. 1907, Astron. Nachr., 175, 325

Breger, M. 1972, ApJ, 171, 539

Breger, M. 1981, ApJ, 249, 666

Breger, M. 2000, ASP Conf. Ser., 210, 3

Breger, M., \& Bischof, K. M. 2002, A\&A, 385, 537

Breger, M., Lenz, P., Antoci, V., et al. 2005, A\&A, 435, 955

Handler, G., O'Donoghue, D., Müller, M., et al. 2003, MNRAS, 340, 1031 
Table 1: Seven Delta Scuti stars that can be studied with BRITE

\begin{tabular}{|c|c|c|}
\hline Star & $\begin{array}{l}\text { Rotation } \\
v \sin i\end{array}$ & Comments, periods, amplitudes \\
\hline$\beta$ Cas & $70 \mathrm{~km} \mathrm{~s}^{-1}$ & $\begin{array}{l}\text { Monoperiodic radial? } \\
\text { near TAMS, } 2.5 \mathrm{~h}, 0.03 \mathrm{mag}\end{array}$ \\
\hline$\rho \operatorname{Pup}$ & $15 \mathrm{~km} / \mathrm{s}$ & $\begin{array}{l}\text { Look for solar-type oscillation, cool, convection } \\
\text { radial pulsation: } 4 \mathrm{~h}, 0.09 \text { mag } \\
\text { single mode or more small-amplitude modes? }\end{array}$ \\
\hline$\gamma$ Boo & $115 \mathrm{~km} / \mathrm{s}$ & $\begin{array}{l}V=3.0, \text { High-degree NRP, } 1 \mathrm{~h} \\
\text { Needs photometric work, doubtful situation, } 0.05 \text { mag? }\end{array}$ \\
\hline$\theta^{2}$ Tau & $77 \mathrm{~km} / \mathrm{s}$ & $\begin{array}{l}\text { Delta Scuti Network, WIRE } \\
\text { well-studied binary (giant and main-sequence) } \\
\text { narrow frequency region - fascinating! }\end{array}$ \\
\hline$\delta$ Ser & $88 \mathrm{~km} / \mathrm{s}$ & $\lambda$ Boo-type spectrum, 4h, $0.05 \mathrm{mag}$ \\
\hline$\nu \mathrm{UMa}$ & $110 \mathrm{~km} / \mathrm{s}$ & $\begin{array}{l}\text { High-degree NRP, } \mathrm{m}=2 \text { to } 11,1.6 \text { to } 2.1 \mathrm{~h} \\
\text { Photometry: maybe } 3 \mathrm{~h} \text { period }\end{array}$ \\
\hline$\rho^{1} \mathrm{Sgr}$ & $82 \mathrm{~km} / \mathrm{s}$ & $\sim 1$ hour, 0.02 mag, F0III-IV \\
\hline
\end{tabular}


Kennelly, E. J., Brown, T. M., Kotak, R., et al. 1998, ApJ, 495, 440

Kilkenny, D., Koen, C., O'Donoghue, D., et al. 1999, MNRAS 303, 525

Lehmann, H., Harmanec, P., Aerts, C., et al. 2001, A\&A, 367, 236

Lenz, P., \& Breger, M. 2005, CoAst 146, 53

Lenz, P., Pamyatnykh, A. A., Breger, M., \& Antoci, V. 2007, A\&A, in press

Petersen, R. C., Carney, B. W., \& Latham, D. W. 1996, ApJ, 465, L47

Zwintz, K., \& Weiss, W. W. 2006, A\&A, 457, 237

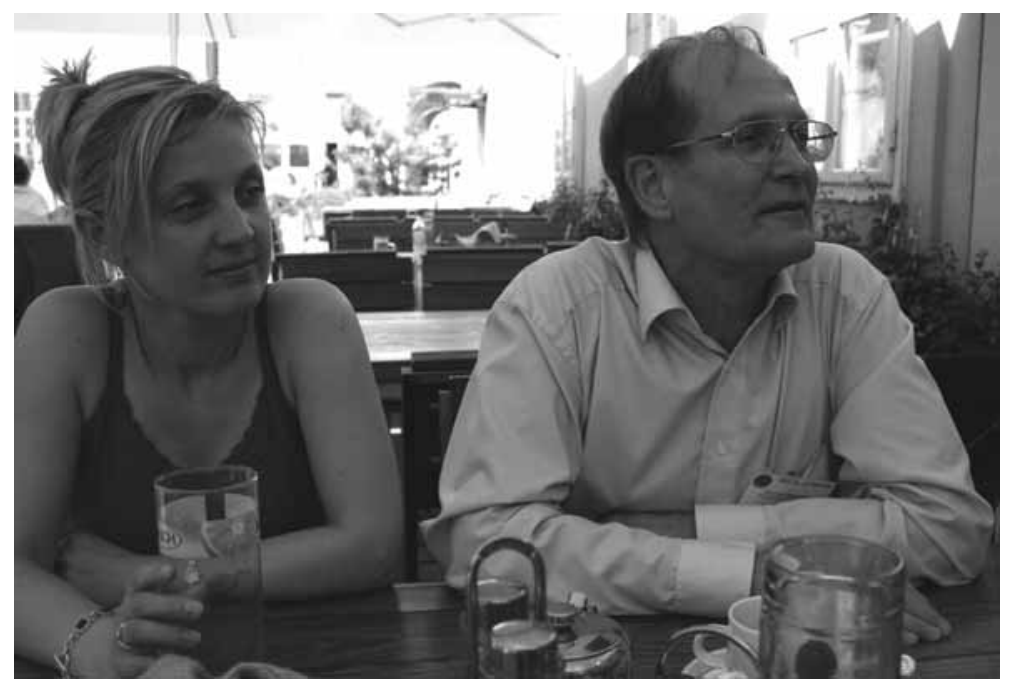

Lunch with J. Daszynska-Daszkiewicz and M. Breger. 